\title{
Assessment of patients with idiopathic inflammatory myopathies and isolated creatin-kinase elevation
}

\author{
L. Iaccarino $\cdot$ E. Pegoraro $\cdot$ L. Bello $\cdot$ \\ S. Bettio $\cdot$ E. Borella $\cdot$ L. Nalotto $\cdot$ C. Semplicini $\cdot$ \\ G. Sorarù $\cdot$ A. Ghirardello $\cdot$ A. Doria
}

Received: 3 September 2014 / Accepted: 9 September 2014/Published online: 15 October 2014

(C) Springer International Publishing Switzerland 2014

\begin{abstract}
Idiopathic inflammatory myopathies (IIM) are a group of diseases characterized by inflammation of the skeletal muscle. Weakness, mainly affecting the proximal muscles, is the cardinal muscular symptom in IIM. In patients with dermatomyositis, peculiar skin lesions are observed. The assessment of patients with IIM includes clinical and laboratory evaluation, and clinimetric measurements. Different tools have been proposed to measure muscular and extramuscular disease activity and damage in patients with IIM. A core set of measurements to use in clinical practice was recently proposed. Among laboratory features the increase of serum creatine kinase $(\mathrm{CK})$ is considered a hallmark of muscle inflammation/damage. However, subjects with persistent CK elevation, without any evidence of a definite myopathy, are often seen in clinical practice and need a careful assessment. Indeed, CK blood levels can also increase in non-myopathic conditions, e.g. in case of intense physical exercise, assumption of some drugs (statins), muscular dystrophy, muscular trauma or in case of neuro-muscular disorders which all should be considered in the diagnostic work-up. The assessment of patients with IIM and hyperCKemia will be discussed in this paper.
\end{abstract}

Keywords Inflammatory myopathies - Polymyositis · Manual muscle test $\cdot$ HyperCKemia

L. Iaccarino - S. Bettio - E. Borella $\cdot$ L. Nalotto ·

A. Ghirardello · A. Doria $(\bowtie)$

Rheumatology Unit, Division of Rheumatology,

Department of Medicine-DIMED, University of Padova,

Via Giustiniani, 2, 35128 Padua, Italy

e-mail: adoria@unipd.it

E. Pegoraro $\cdot$ L. Bello $\cdot$ C. Semplicini $\cdot$ G. Sorarù Department of Neurosciences NPSRR, Neuromuscular Center, University of Padova, Padua, Italy

\section{Introduction}

Idiopathic inflammatory myopathies (IIM) are a group of rare diseases characterized by inflammation of the skeletal muscles. Muscle weakness and increased levels of muscle enzymes are characteristic of these conditions. Four major entities have been identified: polymyositis (PM), dermatomyositis (DM), inclusion body myositis (IBM) and necrotizing myopathies (NM), characterized by focal or diffuse muscular fibers necrosis and scarce inflammation. Other subsets have also been described, including overlap myositis and cancer-associated myositis.

The annual incidence of PM and DM ranges from two to seven cases per million inhabitants with a peak of incidence of 50-60 year. PM and DM are more common in females, with a female: male ratio approximately $2: 1$. The annual incidence of IBM is approximately one-two per million adults and is more common in males [1]. Incidence and prevalence of NM are unknown. PM and DM affect both children and adults; however, juvenile involvement is more frequent in DM. Association with cancer will be discussed in another paper published in this issue.

\section{Assessment of patients with idiopathic inflammatory myopathies}

Clinical features

Muscle involvement

Muscle weakness, mainly affecting the proximal muscles, is the cardinal muscular symptom of patients affected with PM and DM. The onset can be acute (days) or insidious (several months). Myalgias are infrequent. Contractures 
and decreasing strength in the proximal muscles are the most common clinical signs. Muscular atrophy is considered a late sign in the course of the disease and occurs in about $40 \%$ of cases. Respiratory and oropharyngeal muscle involvement must be considered a severe and lifethreatening feature, leading to respiratory difficulties, dysphagia and $a b$ ingestis pneumonia.

Patients with IBM usually have slowly progressive weakness of the distal muscles in the upper limbs, particularly the muscles of the forearm and hand, such as fingers flexors, and proximal muscles, mostly the quadriceps, in the lower limbs. Weakness of distal muscles of lower limbs can cause falls during ambulation. Weakness of other muscles, including that of the swallowing apparatus, although rare, may lead to life-threatening features [2].

\section{Skin involvement}

Gottron's papules (or plaques) and heliotrope rash are the most common and peculiar skin features in patients with DM. Periungual nailfold telangiectasias, cuticular hemorrhage, infarct and/or skin dystrophy are other characteristic skin lesions. Panniculitis, livedo reticularis, non-scarring alopecia, vesiculobullous lesions and a severe generalized erythema, or erythroderma might rarely occur. Calcinosis is usually observed in juvenile DM [1]. Skin manifestations are extensively treated in another article in this issue of the Journal.

\section{Other clinical manifestations}

General symptoms include fever, malaise, weight loss and arthralgias. Raynaud's phenomenon occurs more frequently in IIM associated with other connective tissue diseases. Pulmonary involvement is mainly represented by the interstitial lung pneumonia (ILD) which is characterized by two histological patterns such as non-specific interstitial pneumonia (NSIP) and usual interstitial pneumonia (UIP). NSIP is more frequently observed than UIP in patients with IIM and has better prognosis and good response to immunosuppressive treatment. Heart failure, left ventricular diastolic dysfunction, and hyperkinetic left ventricular contraction may occur. Gastrointestinal involvement is mainly due to disturbed motility and inflammation of the gastrointestinal tract and may result in constipation, diarrhea and stomach pain. GERD (gastroesophageal reflux disease) is common in patients with IIM. Extramuscular organ involvement in patients with IBM is very rare.

Myositis, ILD, Raynaud's phenomenon, non-erosive symmetrical polyarthritis in small joints and scaly skin changes on the hands (mechanic's hands) are associated with anti-synthetase syndrome [3, 4].
Laboratory features

\section{Blood tests abnormalities}

High serum levels of serum creatine kinase (CK) are the hallmark of muscle involvement [5]. CK is released in the serum in case of muscle damage and is the most sensitive muscle enzyme in the acute phase of the disease. In patients with IIM, elevation in serum aldolase, myoglobin, lactate dehydrogenase (LDH), aspartate and alanine aminotransferase (AST and ALT) may also occur. Moreover, serum inflammatory biomarkers (Erythrocyte sedimentation rate, C-reactive protein and others) can also be increased during the active phase of the disease [6].

\section{Autoantibodies}

Autoantibodies associated with IIM are subdivided into myositis-specific autoantibodies (MSA) and myositis associated autoantibodies (MAA), the first occurring only in patients affected with IIM and the latter also in patient affected with autoimmune diseases without myositis [7]. MSA includes anti-Mi-2 antibody associated with classical DM and anti-signal recognition particle (SRP) antibody associated with necrotizing forms [8, 9]. Among other MSA, anti-t-RNA synthetase antibodies are associated with the anti-t-RNA synthetase syndrome [10]. MSA and MAA are reviewed in another paper of this issue of the Journal.

\section{Electromyography}

Although unspecific, abnormalities are observed in up to $90 \%$ of patients underwent needle electromyography. They include increased spontaneous and insertional activity with fibrillation potential, complex repetitive discharges, positive sharp waves, small polyphasic motor units potentials, and early recruitment [1].

\section{Imaging}

Muscle magnetic resonance imaging (MRI) and muscle ultrasound (US) are the two techniques used for the imaging study of muscle diseases. MRI, which is considered the gold standard technique, provides a more detailed anatomic view of the extent of muscle involvement than US and assists the physician in the choice of the muscular biopsy site. Muscle inflammation is identified with MRI by T2-weighted images and short tau inversion recovery (STIR) as muscular oedema. US is considered a good alternative in exploring acute muscle inflammation, especially when MRI is not available. 


\section{Muscular biopsy}

Muscle biopsy is fundamental in the assessment of patients with IIM, and the choice of the correct biopsy site is of key importance. According to the guidelines developed by the International Myositis Assessment and Clinical Study Group (IMACS), a multidisciplinary group of more than 100 experts in adult and juvenile IIM, a positive muscle biopsy is required for a definitive diagnosis of PM [11]. The histological analysis can also distinguish PM from DM, NM and IBM and exclude other myopathies such as dystrophies or metabolic myopathies.

The histologic patterns in patients with IIM are extensively examined in another paper of this issue of the Journal.

Classification of idiopathic inflammatory myopathies

In 1975, Bohan and Peter [12, 13] provided a classification for patients with IIM which included four muscular criteria and one cutaneous criterion (Table 1). Accordingly, patients were classified as affected with IIM only after the exclusion of other diagnoses. Despite the high sensitivity $(94.3 \%)$, Bohan and Peter's criteria show a low specificity (29.4\%). Indeed, these criteria are not able to clearly distinguish DM and PM from IBM or other myopathies, particularly, dystrophies [5, 14]. Thus, it has been suggested to add MSA and MRI to increase their specificity $[15,16]$. Despite this weakness, "probable" or "definite" PM and DM, as defined by Bohan and Peter's criteria, are still used in clinical trials, according to the consensus guidelines developed by IMACS [11].

Table 1 Bohan and Peter's polymyositis and dermatomyositis diagnostic criteria

1. Symmetric proximal muscle weakness determined by physical examination

2. Elevation of serum skeletal muscle enzymes, including CK, aldolase, serum glutamate oxaloacetate and pyruvate transaminases, and lactate dehydrogenase

3. The electromyographic triad of short, small, polyphasic motor unit potentials; fibrillations, positive sharp waves, and insertional irritability; and bizarre, high-frequency repetitive discharges

4. Muscle biopsy abnormalities of degeneration, regeneration, necrosis, phagocytosis, and an interstitial mononuclear infiltrate

5. Typical skin rash of DM. Including a heliotrope rash and Gottron's sign/papules

The diagnosis of polymyositis is considered defined, probable and possible when 4,3 , or 2 muscle criteria are present, respectively

The diagnosis of dermatomyositis is considered definite, probable and possible when skin rash is associated with 3,2 , or 1 muscular criterion, respectively

Exclusion criteria: central or peripheral neurologic diseases, muscular dystrophies, granulomatous and infectious myositis, metabolic and endocrine myopathies, and myasthenia gravis
In 2003, Dalakas and Hohfeld [5] proposed a set of muscle biopsy-based criteria. In this classification, PM and IBM were differentiated from other muscular diseases, particularly dystrophies, by using MHC-1/CD8 complex as a specific marker of immune activation. The specificity of Dalakas and Hohfeld's criteria is very high (99.9\%), probably because of their precise definition of the histological findings associated with PM and DM. However, the sensitivity is $77.1 \%$ [14]. Indeed, in the case of a poorly informative muscle biopsy, clinicians may have difficulties in differentiating PM and DM from other muscle disorders [17]. MSA and MRI findings, useful in the differential diagnosis, were not considered in these criteria. Indeed, muscle biopsy is sometimes not necessary, for instance in DM cases with specific skin manifestations, such as Gottron's papules and heliotrope rash.

The classification proposed by the European Neuromuscular Centre (ENMC) is based on clinical features, laboratory findings (including MSA), muscular MRI and muscle biopsy. Inclusion and exclusion criteria have both to be satisfied to classify patients as affected with IIM [18]. The inclusion of MSA and precise hystological definitions are the strength of these criteria [1], but their use in clinical practice is hampered by their complexity. Specificity is $82.4 \%$ and sensitivity $71.4 \%$ [14]. Moreover, overlap syndromes with IIM are not included in this classification.

\section{Clinimetric measurements}

In clinical practice, distinguishing clinical features due to disease activity from those related to damage is an essential part in the assessment of patients affected with IIM.

Disease activity was recently defined as "extent and severity of reversible manifestations due to muscular and extra-muscular disease", whereas damage was defined as "persistent changes in anatomy, physiology, pathology or function resulting from prior active disease, complications of therapy, co-morbid conditions, which do not contribute to an active disease process and reflects scarring, atrophy and fibrosis" [19]. Disease activity is reversible with therapy, whereas damage is often irreversible and cumulative [19].

Several measures of activity and damage have been developed and proposed during recent years [20]. Recently, the IMACS defined a consensus core set of measures (Table 2) to assess disease activity and damage. It is used in clinical practice and in randomized controlled trials [19].

\section{Disease activity assessment tools}

The Physician and Patient/Parent Global Activity (PGA) assessment obtained from the physician and patient, who are asked to consider all the active inflammatory lesions of 
Table 2 Disease activity and damage: IMACS core set measurements

\author{
Disease activity \\ Physician global activity_VAS/Likert \\ Patient/parent global activity-VAS/Likert \\ Muscle strength-manual muscle test (MMT) \\ Physical function- $[\mathrm{C}] \mathrm{HAQ}$, CMAS \\ Laboratory-muscle enzymes \\ Extra-muscular activity—MDAAT \\ Damage \\ Myositis Damage Index (MDI) \\ Physician global damage-VAS/Likert \\ Physical function- $[\mathrm{C}] \mathrm{HAQ}$
}

$C$ childhood, CMAS Childhood Myositis Assessment Scale, VAS visual analog scale, $H A Q$ health assessment questionnaire, MDAAT myositis disease activity assessment tool, IMACS International Myositis Assessment and Clinical Studies Group

muscles, skin, joints, heart, lungs and other parts of the body [20], and to record the score on a 10-cm visual analog scale (VAS) or a 5-point rating, the Likert scale. Score zero correspond to no disease activity and score four (in the Likert scale) or ten (in the VAS scale) to extremely severe disease activity. The 10-cm VAS has better precision, sensitivity, and specificity than 0-4 Likert scale; however, the two scales are highly correlated.

Manual muscle testing (MMT) measures muscle strength and is part of physical examination [19, 21]. The number of muscle groups considered in the score is not standardized. Recently, a subset of eight muscle groups, including neck flexors, deltoids, biceps, wrist extensors, gluteus maximus (Fig. 1) and medius, quadriceps, and ankle dorsiflexors has been proposed and validated by the IMACS group [22]. The final score is the sum of scores of the eight muscle groups. Maximum score is 150 if proximal and distal muscles are tested bilaterally and 80 if they are tested unilaterally (neck flexors can only be tested unilaterally). Both the modified Medical Research Council
(MRC) muscle strength scale (Table 3) and the Kendall grading scale are used to score the strength of muscles [19, 21]. MMT is a valid measure of muscular strength; however, it does not discriminate between disease activity and damage, resulting in less sensitivity and specificity for patients with damage accrual and progressive muscle atrophy.

Myositis disease activity assessment tool (MDAAT) is a combined tool that assesses muscle disease activity and extramuscular organ systems. It consists of two subscales: the myositis disease activity assessment (MYOACT) and the Myositis Intention to Treat Activities Index (MITAX), both scoring disease activity in seven organs/systems: constitutional, muscle, skeletal, cutaneous, pulmonary, cardiac and gastrointestinal. The MYOACT use the 0-10 VAS scale. The MITAX is based on an intention-to-treat approach modified from the British Isles Lupus Assessment Group (BILAG) used for patients with systemic lupus erythematosus [23]. Clinical features are recorded using a scale of $0-4(0=$ not present, $1=$ improving, $2=$ the same, $3=$ worse and $4=$ new). This score is converted to a final score ranging from $\mathrm{A}$ to $\mathrm{E}$ (Table 4). The MDAAT provides disease activity in various organ systems and it is useful for patients with extramuscular involvement; a trained physician is required for the assessment.

\section{Disease damage assessment tools}

Myositis Damage Index (MDI) measures both severity and extent of damage in 11 organ systems: muscular, cutaneous, skeletal, gastrointestinal, pulmonary, cardiovascular, peripheral vascular, endrocrine, ocular, malignancy, and infections. Eleven separate VAS (0-10) constitute the MDI severity of damage scale. Zero is scored in case of absolute absence of damage and 10 in case of extremely severe damage. Each system has also 3-6 items to be measured as present or absent (score 0 or 1 , respectively). A positive score is assessed if the item is present for at least 6 months despite prior immunosuppressive or other therapy.
Fig. 1 Example of MMT: evaluation of quadriceps. a Antigravity position, $\mathbf{b}$ gravity eliminated position
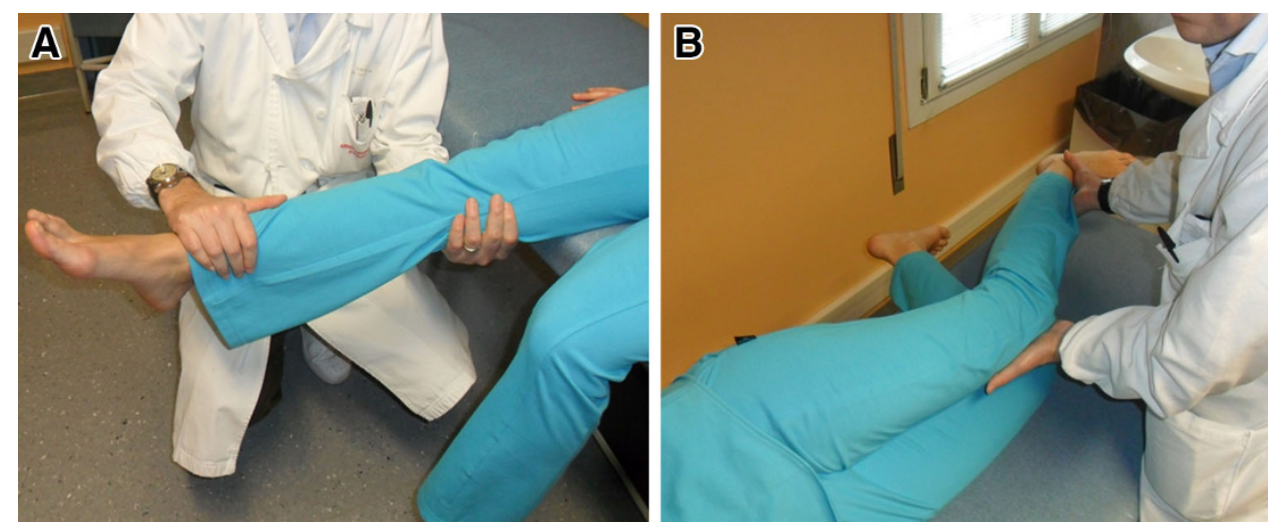
Table 3 MRC Muscle Strength Scale

\begin{tabular}{|c|c|c|}
\hline Grade & Muscle function & Plane of movement \\
\hline 0 & No contraction & - \\
\hline $\mathrm{T}$ & $\begin{array}{l}\text { Tendons become prominent or } \\
\text { feeble contraction felt in the } \\
\text { muscle, but not visible } \\
\text { movement of the part }\end{array}$ & - \\
\hline 1 & $\begin{array}{l}\text { Moves through partial range of } \\
\text { motion }\end{array}$ & $\begin{array}{l}\text { Horizontal plane } \\
\text { (gravity eliminated } \\
\text { position) }\end{array}$ \\
\hline 2 & $\begin{array}{l}\text { Moves through complete range of } \\
\text { motion }\end{array}$ & $\begin{array}{l}\text { Horizontal plane } \\
\text { (gravity eliminated } \\
\text { position) }\end{array}$ \\
\hline 3 & $\begin{array}{l}\text { Movement trough partial range of } \\
\text { motion }\end{array}$ & Antigravity position \\
\hline 4 & Gradual release from test position & Antigravity position \\
\hline 5 & $\begin{array}{l}\text { Holds test position without added } \\
\text { pressure }\end{array}$ & Antigravity position \\
\hline 6 & $\begin{array}{l}\text { Hold test position against slight } \\
\text { pressure }\end{array}$ & Antigravity position \\
\hline 7 & $\begin{array}{l}\text { Hold test position against slight to } \\
\text { moderate pressure }\end{array}$ & Antigravity position \\
\hline 8 & $\begin{array}{l}\text { Hold test position against moderate } \\
\text { pressure }\end{array}$ & Antigravity position \\
\hline 9 & $\begin{array}{l}\text { Hold test position against moderate } \\
\text { to strong pressure }\end{array}$ & Antigravity position \\
\hline 10 & $\begin{array}{l}\text { Holds test position against strong } \\
\text { pressure }\end{array}$ & Antigravity position \\
\hline
\end{tabular}

Table 4 MITAX scoring schema

\begin{tabular}{ll}
\hline A & $\begin{array}{r}\text { Very active disease requiring treatment with high dose daily } \\
\text { corticosteroids or a significant immunosuppressive therapy }\end{array}$ \\
B $\quad \begin{array}{c}\text { Need for modest doses of corticosteroids and/or ongoing } \\
\text { immunosuppression }\end{array}$ \\
C $\quad$ Need for low-dose steroid or symptomatic drugs only \\
D $\quad$ The system is no longer active \\
E
\end{tabular}

Unfortunately, it does not measure only damage related to disease, but it also captures other pathological conditions.

\section{Assessment of patients with isolate increase of creatine kinase}

$\mathrm{CK}$ is an enzyme which catalyzes the conversion of creatine into phosphocreatine. In the reaction one molecule of adenosine triphosphate (ATP) is consumed, and turned into adenosine diphosphate (ADP). This reaction is reversible, allowing the storage of readily expendable energy under the form of phosphocreatine, especially in tissues with high metabolic demands, leading to rapid consumption of ATP, such as skeletal muscle, retinal or cochlear sensory receptors, or brain. The human genome harbors five CK genes, encoding two cytosolic isoforms (CK-M, for "muscle", and CK-B, for "brain"), and three mitochondrial isoforms. CK-M and CK-B monomers combine to form enzymatically active homo- or heterodimers: CKMM (90\% of CK in skeletal muscle), CK-MB (40\% in the myocardium), and CK-BB (96\% in the brain). Mitochondrial CK subunits assemble into both dimers and octamers, and localize in the space between the inner and outer mitochondrial membranes.

\section{Definition of hyperCKemia}

HyperCKemia may be defined as the persistent elevation of plasma CK activity levels. Normal ranges of plasma CK activity vary depending on different physiological conditions such as gender, ethnicity, age, muscle mass, and physical exercise, and have been set at different values by different authors. A recent study determined that as many as $13 \%$ of healthy Caucasians, and up to $49 \%$ of healthy people of African descent may present elevated CK [24]. In the same study, the 97.5th percentile for normal values consistently varied based on gender and ethnicity.

Persistent elevation is usually determined by confirming the elevated value in repeated samples, possibly at least 3 weeks apart, observing at least $72 \mathrm{~h}$ of rest before sample collection $[25,26]$.

\section{Causes of elevated creatine kinase plasma activity}

Contraction of muscle fibers, especially if the fibers are lengthened during contraction (eccentric exercise), causes mechanical damage to sarcoplasmatic membranes, increasing their permeability, and leading to leakage of cytoplasmic enzymes into the extracellular fluid and the bloodstream. Conditions causing an elevation of plasma CK activity beyond normal levels might be related to a specific, recognizable primary disease of skeletal muscle, or alternatively caused by different mechanisms. Some patients with persistently elevated $\mathrm{CK}$, for whom even extensive clinical and laboratory studies fail to identify a cause, fall under the nosographic category of idiopathic hyperCKemia, coined by Rowland et al. [27] in 1980.

HyperCKemia unrelated to a recognized neuromuscular disease

Non-primitively muscular causes of CK elevation should be excluded first, when approaching a patient with isolated hyperCKemia. Exercise, especially when involving strenuous and eccentric muscle contraction, which damages skeletal muscle fiber structure at the level of sarcolemma and Z-disks, is perhaps the most common cause. After 
strenuous exercise, CK levels peak at $8 \mathrm{~h}$ and remain elevated around $24 \mathrm{~h}$; with eccentric contractions, the peak is reached more slowly $(96 \mathrm{~h}$ ) and it may take more than a week to revert to normal levels. Persistent hyperCKemia should be confirmed after remaining sedentary at least $72 \mathrm{~h}$. Even resting levels are higher in athletes than sedentary subjects.

Other non-neuromuscular causes of elevated CK may be iatrogenic, toxic, metabolic (hypothyroidism, hypoparathyroidism), or related to viral infection, heart disease (with preferential elevation of CK-MB), or other specific, sometimes rare, conditions (e.g. obstructive sleep apnea syndrome, malignant hyperthermia, neuroacanthocytosis).

Drug-induced hyperCKemia is common in clinical practice, and most frequently lipid-lowering agents are the culprit. Statins, i.e. inhibitors of 3-hydroxy-3-methylglutarylcoenzyme A (HMG-CoA) reductase, not only limit the synthesis of cholesterol, but also the farnesylation and prenylation of several proteins including lamin $\mathrm{A} / \mathrm{C}$, selenoprotein $\mathrm{N}$, and sarcoglycans, and the biosynthesis from mevalonic acid of cofactors such as Coenzyme Q. These mechanisms jointly destabilize the sarcolemma and impair the mitochondrial redox potential [28]. While more severe conditions, like rhabdomyolysis or statin myopathy with clinically evident weakness, are relatively rare, isolated
hyperCKemia and myalgia are common, affecting up to one-third of patients in some clinical practice case series. Other drugs frequently associated to elevation of $\mathrm{CK}$ are fibrates, colchicine, anti-psychotic drugs (including neuroleptic malignant syndrome), zidovudine, and beta blockers. Alcohol, heroin, and cocaine intoxications all may cause elevation of CK.

In association with autoimmune disease or malignancy, CK clearance and inactivation might be impaired, respectively because of association with immune complexes, or because of aggregation of high molecular mass mitochondrial CK. These conditions are known as "macro CK" and cannot be distinguished from other forms of hyperCKemia by standard CK activity assays.

Finally, trauma and invasive medical acts such as intramuscular injection, needle electromyography, and surgery may lead to elevated CK.

HyperCKemia related to a recognized neuromuscular disease

In primitive neuromuscular disease, elevated CK is often associated with specific signs and symptoms (primarily muscular weakness) and represents a useful diagnostic biomarker. It should be remembered that hyperCKemia is

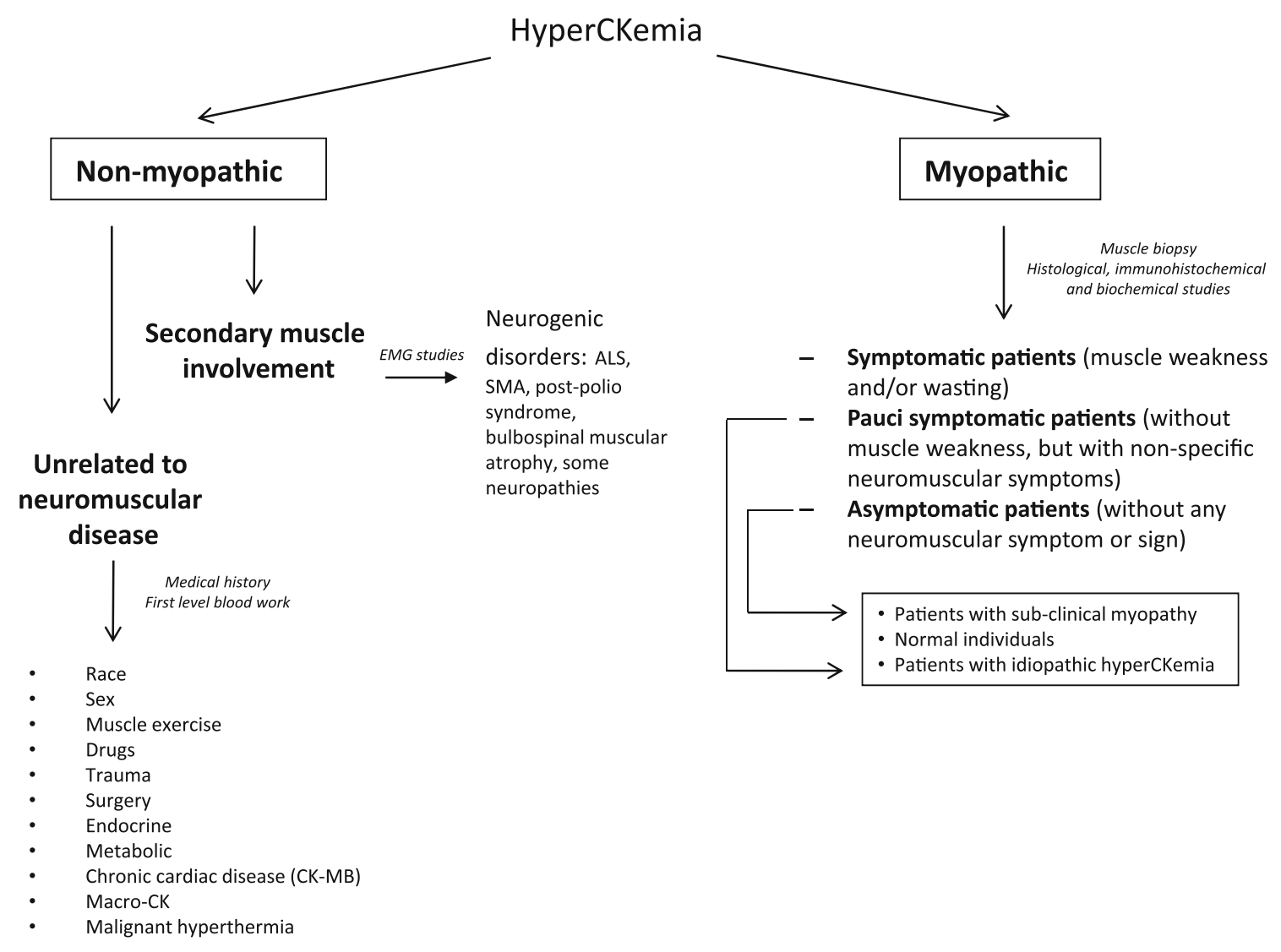

Fig. 2 Diagnostic work-up of hyperCKemia 
reported not only in myopathic syndromes, but also in some neurogenic conditions such as amyotrophic lateral sclerosis, hereditary spinal muscular atrophy, post-polio syndrome, bulbo-spinal muscular atrophy, and some neuropathies [29].

Nevertheless, several neuromuscular diseases may present with isolated elevation of plasma CK in the absence of specific neuromuscular signs and symptoms. These most commonly include: adult onset glycogenosis type 2, caveolinopathy (caveolin-3), calpainopathy (calpain-3), desminopathy, dysferlinopathy (LGMD and Miyoshi phenotypes), fukutin-related protein (FKRP) (LGMD2I), dystrophinopathy (mainly female carriers and Becker Muscular Dystrophy), sarcoglycanopathy, myotonic dystrophy type 2, and calsequestrin aggregate myopathy [29, 30]. A case series of 2014 patients with isolated hyperCKemia who underwent a muscle biopsy at University of Padova showed that, in $86 \%$ of cases, the diagnosis of a specific genetic neuromuscular disorder was not reached, despite extensive histological, immunohistochemical and biochemical studies [31] (Fig. 2).

\section{Clinical approach and guidelines}

The clinical approach (Fig. 2) to patients with an isolated finding of elevated CK starts with the exclusion of "physiological" and non-neuromuscular causes, primarily by establishing a persistent elevation with repeated measurements at rest, possibly in the absence of CKelevating medications. When a persistent elevation of $\mathrm{CK}$ is established, electromyography has a critical role in differentiating myopathic vs. neurogenic disorders, and further selecting patients for muscle biopsy. Detailed guidelines with criteria for selecting patients with isolated/asymptomatic CK for muscle biopsy have been issued by panels of experts from both the Italian Association of Myology [32], and the European Federation of Neurological Societies [29]. These take into account the degree of elevation of CK, gender (with particular attention to the importance of correctly identifying Duchenne Muscular Dystrophy carriers of childbearing age), electrophysiological findings, and psychological concern or distress caused by the finding.

In conclusion, in cases of isolated finding of elevated CK, a correct clinical and laboratory work-up benefits patients, not only by ensuring a prompt diagnosis, potentially preventing morbidity in the patient and his relatives, and enabling physicians to adjust medical therapies, but also by avoiding the clinical and economic burden related to unnecessary medical tests.

Conflict of interest Authors declare that they have no conflict of interests
Human and animal rights All procedures performed in studies involving human participants were in accordance with the ethical standards of the institutional and/or national research committee and with the 1964 Helsinki declaration and its later amendments or comparable ethical standards.

\section{References}

1. Iaccarino L, Ghirardello A, Bettio S, Zen M, Gatto M, Punzi L, Doria A (2014) The clinical features, diagnosis and classification of dermatomyositis. J Autoimmun 48-49:122-127

2. Briani C, Doria A, Sarzi-Puttini P, Dalakas MC (2006) Update on idiopathic inflammatory myopathies. Autoimmunity 39:161-170

3. Iaccarino L, Gatto M, Bettio S, Caso F, Rampudda M, Zen M, Ghirardello A, Punzi L, Doria A (2013) Overlap connective tissue disease syndromes. Autoimmun Rev 12:363-373

4. Hervier B, Devilliers H, Stanciu R et al (2012) Hierarchical cluster and survival analyses of antisynthetase syndrome: phenotype and outcome are correlated with anti-tRNA synthetase antibody specificity. Autoimmun Rev 12:2010-2017

5. Dalakas MC, Hohlfeld R (2003) Polymyositis and dermatomyositis. Lancet 362:971-982

6. Yuan L, Yao L, Zhao L, Xia L, Shen H, Lu J (2013) Serum levels of soluble ST2 and interleukin-33 in patients with dermatomyositis and polymyositis. Clin Exp Rheumatol 31:428-432

7. Ghirardello A, Bassi N, Palma L, Borella E, Domeneghetti M, Punzi L, Doria A (2013) Autoantibodies in polymyositis and dermatomyositis. Curr Rheumatol Rep 15:335

8. Hengstman GJ, ter Laak HJ, Vree Egberts WT, Lundberg IE, Moutsopoulos HM, Vencovsky J, Doria A, Mosca M, van Venrooij WJ, van Engelen BG (2006) Anti-signal recognition particle autoantibodies: marker of a necrotizing myopathy. Ann Rheum Dis $65: 1635-1638$

9. Ghirardello A, Zampieri S, Iaccarino L, Tarricone E, Bendo R, Gambari PF, Doria A (2005) anti-Mi-2 antibodies. Autoimmunity 38:79-83

10. Zampieri S, Ghirardello A, Iaccarino L, Tarricone E, Gambari PF, Doria A (2005) Anti-Jo-1 antibodies. Autoimmunity 38:73-78

11. Oddis CV, Rider LG, Reed AM et al (2005) International consensus guidelines for trials of therapies in idiopathic inflammatory myopathies. Arthritis Rheum 52:2607-2615

12. Bohan A, Peter JB (1975) Polymyositis and dermatomyositis (first part). N Engl J Med 292:344-347

13. Bohan A, Peter JB (1975) Polymyositis and dermatomyositis (second part). N Engl J Med 292:403-407

14. Linklater H, Pipitone N, Rose MR, Norwood F, Campbell R, Salvarani C, Scott DL, Gordon P (2013) Classifying idiopathic inflammatory myopathies: comparing the performance of six existing criteria. Clin Exp Rheumatol 31:767-769

15. Targoff IN, Miller FW, Medsger TA, Oddis CV (1997) Classification criteria for idiopathic inflammatory myopathies. Curr Opin Rheumatol 9:527-535

16. Troyanov Y, Targoff IN, Tremblay JL, Goulet JR, Raymond Y, Senecal JL (2005) Novel classification of idiopathic inflammatory myopathies based on overlap syndrome features and autoantibodies: analysis of 100 French Canadian patients. Medicine 84:231-249

17. Miller FW, Rider LG, Plotz PH, Isenberg DA, Oddis CV (2003) Diagnostic criteria for polymyositis and dermatomyositis. Lancet 362:1762-1763

18. Hoogendijk JE, Amato AA, Lecky BR et al (2004) 119th ENMC international workshop: trial design in adult idiopathic inflammatory myopathies, with the exception of inclusion body myositis, 10-12 october 2003, Naarden, The Netherlands. Neuromuscul Disord 14:337-345 
19. Miller FW, Rider LG, Chung YL et al (2001) Proposed preliminary core set measures for disease outcome assessment in adult and juvenile idiopathic inflammatory myopathies. Rheumatology 40:1262-1273

20. Rider LG, Werth VP, Huber AM, Alexanderson H, Rao AP, Ruperto N, Herbelin L, Barohn R, Isenberg D, Miller FW (2011) Measures of adult and juvenile dermatomyositis, polymyositis, and inclusion body myositis. Arthritis Care Res 63:S118-S157

21. Rider LG, Giannini EH, Harris-Love M et al (2003) Defining clinical improvement in adult and juvenile myositis. J Rheumatol 30:603-617

22. Rider LG, Koziol D, Giannini EH et al (2010) Validation of manual muscle testing and a subset of eight muscles for adult and juvenile idiopathic inflammatory myopathies. Arthritis Care Res 62:465-472

23. Hay EM, Bacon PA, Gordon C, Isenberg DA, Maddison P, Snaith ML, Symmons DP, Viner N, Zoma A (1993) The BILAG index: a reliable and valid instrument for measuring clinical disease activity in systemic lupus erythematosus. Q J Med 86:447-458

24. Brewster LM, Mairuhu G, Sturk A, van Montfrans GA (2007) Distribution of creatine kinase in the general population: implications for statin therapy. Am Heart J 154:655-661

25. Prelle A, Tancredi L, Sciacco M et al (2002) Retrospective study of a large population of patients with asymptomatic or minimally symptomatic raised plasmacreatine kinase levels. J Neurol 249:305-311
26. Simmons Z, Peterlin BL, Boyer PJ, Towfighi J (2003) Muscle biopsy in the evaluation of patients with modestly elevated creatine kinase levels. Muscle Nerve 27:242-244

27. Rowland LP, Willner J, DiMauro S, Miranda A (1980) Approaches to the membrane theory of Duchenne muscular dystrophy. In: Angelini C, Danieli GA, Fontanari D (eds) Muscular dystrophy-advances and new trends. Excerpta Medica, Amsterdam, pp 3-13

28. Vaughan CJ, Gotto AM Jr (2004) Update on statins: 2003. Circulation 17(110):886-892

29. Kyriakides T, Angelini C, Schaefer J et al (2010) EFNS guidelines on the diagnostic approach to pauci- or asymptomatic hyperCKemia. Eur J Neurol 17:767-773

30. Rossi D, Vezzani B, Galli L et al (2014) A mutation in the CASQ1 GeneCauses a vacuolar myopathy with accumulation of sarcoplasmic reticulum proteinAggregates. Hum Mutat. doi:10. 1002/humu.22631

31. Fanin M, Nascimbeni AC, Aurino S, Tasca E, Pegoraro E, Nigro V, Angelini C (2009) Frequency of LGMD gene mutations in Italian patients with distinct clinical phenotypes. Neurology 21(72):1432-1435

32. Morandi L, Angelini C, Prelle A, Pini A, Grassi B, Bernardi G, Politano L, Bruno C, De Grandis D, Cudia P, Citterio A (2006) High plasma creatine kinase: review of the literature and proposal for a diagnostic algorithm. Neurol Sci 27:303-311 